\title{
LBNL Report of the Vetting Review of the GRETINA Project
}

\section{The GRETINA Vetting Review was held on Nov. 4 \& 5, 2003}

\author{
Date of this Report: Jan. 12, 2004
}

with Addendum of Mar. 29, 2004

\author{
J. H. Bercovitz, F. S. Bieser, R. C. Jared, V. P. Karpenko, S. R. Klein, K. T. Lesko, J. E. Rasson, \\ H. G. Ritter, K. E. Robinson (chair), C. E. Tull, R. Wells, H. H. Wieman \\ E. O. Lawrence Berkeley National Laboratory
}

\section{Review Scope and Charge}

GRETINA is a gamma-ray detector array capable of reconstructing the energy and spatial positions of gamma-ray interactions within the germanium crystals. It will be used to study the structure and stability of nuclei under various conditions. The new capabilities provided by gamma-ray tracking will give large gains in sensitivity for a large number of experiments, particularly those aimed at nuclei far from beta stability.

A proposal for GRETINA was submitted to DOE in June 2003. It presented the scientific case, the readiness of technical development, the design, the suggested management organizations, and a proposed cost and schedule. The GRETINA proposal received its CD0 approval in August 2003. The CD-1 review will be held on December 3 and 4, 2003, and will be handled by the DOE-N.

This report presents the charge to the GRETINA vetting review committee, and the findings, comments and recommendations of this committee.

The purpose of this project vetting review was to assure that the GRETINA project is on track to provide DOE and the nuclear physics community with the agreed upon deliverables within the agreed upon budget and schedule. The vetting review committee was asked to cover both technical and management aspects of the GRETINA Project. Reviewers offer expert knowledge in relevant areas and provide recommendations and findings to the project's management team. Upon successful completion, the Laboratory's Integrated Project Management Office (IPMO) will recommend signoff of the project to the Laboratory Directorate.

The GRETINA vetting review committee was asked to consider all relevant aspects of the project's management, project execution plan (PEP), technical approach and status, cost estimate, resources, schedule and risk and, in doing so, to advise as to whether the GRETINA Project was likely to successfully provide the agreed upon deliverables within the agreed upon budget and schedule.

The review committee was asked to identify any project areas that may be incomplete for the current phase in the project and any area of significant risk for the project reaching its objectives. The 
committee was also asked to review and evaluate the technical status of the project and advise on any concerns or significant technical risks.

Specific questions that the committee was asked to answer (and that were expected to frame the content of the information provided by the project to the reviewers) included the following questions.

1. Does the project have an effective organizational structure? Are the project roles and responsibilities well defined and understood by those who carry out the responsibilities? Are tasks, responsibilities, and authority properly delegated?

2. Are the cost and schedule for the project well understood and are they consistent with the overall funding and schedule for the project?

3. Is the schedule resource loaded, comprehensive and verifiable in appropriate detail? Are schedule milestones clearly identified, and are the milestones frequent enough to gauge progress? Does the schedule specify relationships, critical paths, slack paths, and resources in appropriate detail for a project of this size?

4. Is the project plan appropriate, is it an effective tool to guide the project to completion, and is it being used effectively by the project leadership? Does the project plan include relevant portions appropriate to a project of this size, such as the Statement of Work (SOW), Work Breakdown Structure (WBS), Risk Management Plan, and the Budget and Schedule Estimates?

5. Are the technical requirements well understood and documented? Does the baseline design meet the project's objectives? Has adequate R\&D been completed in a timely manner to support the construction goals of the project?

6. Is the level of technical progress appropriate for this stage of the project? Are there any potential technical showstoppers or major uncertainties? Are there adequate resources (people, funds, infrastructure) to support the technical goals?

7. Does the DOE expect LBNL to provide facilities, special test equipment, and/or resources that are not part of the project's cost baseline?

8. Is the involvement of other institutions appropriate, well understood and needed for the successful completion of GRETINA? Are there any appropriate written agreements such as MOUs that fully describe the commitments and deliverables of other institutions to GRETINA (including schedules, important interfaces, and requirements) as well as any resources to be provided from the GRETINA project and/or from LBNL that are needed to meet these commitments? Is it likely that the other institutions will successfully deliver as planned?

9. Is there a QA plan that is realistic and appropriate for a project of this scale? 


\section{Findings}

\section{Detector and Electronics}

Technically the project is in excellent shape. The review was a very impressive demonstration of an innovative detector approach. The ability to de-convolute multiple hits in the detector with $1 \mathrm{~mm}$ resolution is quite remarkable. The presentation made it clear that this detector makes a truly significant advance in gamma ray measurements.

\section{Data Acquisition and Computing}

The algorithmic approach to finer position resolution in segmented Ge detectors is an impressive accomplishment.

There appear to be no insurmountable technical hurdles in the computing domain, as most data volume and processing requirements are modest compared to reasonable projections of hardware capabilities and costs at the time of procurement.

\section{Mechanical Systems}

The EDI cost fraction of 50\% seems reasonable.

The mechanical system appears well within the capabilities of the GRETINA collaboration.

\section{Project Management}

The GRETINA project team did a good job in putting together the PEP.

Management roles and responsibilities within the GRETINA project are not fully defined and worked out. Specifically, it is not clear what areas are the responsibility of the Project Director as opposed to the Project Manager. This should be clarified.

The cost of purchasing the crystals alone is about $\$ 5 \mathrm{M}$ of the project total, and could involve justifying a sole source purchase. This is a serious procurement and cost issue. Furthermore, the review committee was concerned that a large order for crystals might be placed by a coincident European project. Does the vendor have the production capability to fulfill two large orders for crystals during the next five or six years?

The review committee was concerned about the mismatch between the schedule and the funding profile.

There are no high-level flow-down requirements.

The review committee was concerned about the lack of clarity in the definition of project completion criteria. 
The scheduling of the CD gates and the schedule do not mesh.

There is a two-year hole in the mechanical engineering effort, with the issue of mothballing and starting up again.

The issue of interface specifications documents is not fully addressed.

The project will adopt an earned value process.

The Project Director, I.-Yang Lee, should control every dollar of the contingency.

A descoping plan has not been developed to address contingency management. While it might be tempting to think of the number of detectors as a contingency of last resort, descoping the number of detectors could compromise the scientific value of GRETINA. What is the minimum threshold for a scientifically viable detector (using the number of detectors as scope contingency of last resort)?

DOE may have some flexibility in the funding profile. With a slightly different funding profile, the risk with the detectors could decrease significantly. The sooner that DOE commits to the funding profile and the sooner the order is placed for the long lead detectors, the lower the risk.

Barry Savnik is currently writing the Acquisition Strategy.

\section{Comments}

\section{Detector and Electronics}

The collaboration should develop a set of interface documents, detailing the connections between various modules, data formats, control signals, etc. These documents should be placed under change control.

EPICS is a good choice for control software. It is worth expending some effort to try to make sure that the different subsystems, such as the liquid nitrogen controller, all have EPICS interfaces.

The collaboration should examine the requirements for cooling and fire safety for the electronics racks.

The collaboration should write a formal requirements document for the electronics, detailing the need for the electronics performance requirements. The collaboration has already done most of the needed evaluations; these findings should be written down.

The current trend in electronics is away from VME and toward compact PCI (cPCI). There is a much wider selection of cPCI CPU boards than VME based boards, and Cypress no longer makes VME interface chips. The committee suggests that the collaboration consider using $\mathrm{cPCI}$ for the next version of the DSP board (and trigger boards). 
The collaboration should ensure that the numbers are consistent between various documents.

The plan is to thoroughly map one detector of each type and to extrapolate to the other detectors, possibly with spot checks of the other detectors. Issues of ageing and radiation damage may need to be considered, and may be non-trivial. They have done a very good job on the mapping of the detector so far.

Calibrations and evaluations of the detectors do not show enough physicist involvement.

\section{Data Acquisition and Computing}

EPICS is clearly a good choice for experiment control of thresholds and other hardware and electronics parameters. It is less clear that using EPICS to control the parameters for processes running on the online Decomposition Farm is the best choice. Other options for the decomposition algorithm processes should be considered, including:

a) Use of software similar to the Event Feeder.

b) Use of a commercial or open-source DataBase.

c) Use of a shared file system and globally accessible configuration files.

The approach of "Physicist develops algorithm, engineer implements" is unlikely to work. The recipe for success is for developers of Physics-related software to take long-term ownership of algorithms. This implies that developers of such software must take responsibility for production quality of code (including issues such as robustness, performance and resource utilization) and have commensurate computing knowledge and experience. Engineers or computing professionals should provide an infrastructure and/or a framework in which scientists develop software.

The cost per CPU of the Decomposition Farm seems a little low. It seems to reflect hardware costs, but no construction/installation costs. The conservative estimates of the effects of Moore's Law used may offset this.

The event processing data flow has several points where some failure rate is to be expected:

a) The Readout CPU module buffer can fill with no available nodes in the Decomposition Farm, thus discarding subsequent events until resolved. This is a consequence of the push model and finite buffer size for the module.

b) Hardware and software failures are to be expected on the Farm nodes.

c) The event collector may timeout without all event components being available. Careful handling of partial events is needed. Bookkeeping of upstream failures is required. The output data stream needs to flag partial events for offline physics analysis. This information affects the overall efficiency of the detector system.

In addition to expected failures, it is anticipated that the analysis processes on the Farm nodes will have a maximum productive lifetime:

a) Farm monitoring requirements are needed that can be used in the procurement of the Farm. It may be possible to procure a commercially/professionally assembled farm with integrated monitoring. However, the requirements from the GRETINA side should be understood to assess whether such a system is sufficient. Current best practices involve 
Ganglia-like farm monitoring and application monitoring with Grid-like tools such as NetLogger.

b) Estimates of the expense of setup and teardown for a farm process relative to the event processing cost will dictate the frequency with which a cyclic stop/restart should be done.

\section{Mechanical Systems}

Why does the cost summary show redirected effort of $\$ 75 \mathrm{k}$ for Supports and $\$ 50 \mathrm{k}$ for Target?

The review committee had the following concerns about the ORNL liquid Nitrogen system:

a) The cost of the computer control system and the electronics seems high.

b) Is this a GRETA-compatible system or a GRETINA only system?

c) Has ORNL agreed to a 2007 start? What assurance does the project have that the resources will be available?

For the mechanical aspects, a 3D model can be used to assure that everything fits. The control and revision of the model requires thought and deliberate action. The model may not be able to address issues of dimensional tolerances and assembly.

\section{Project Management}

The Management Advisory Committee has no representative from Engineering. (There are representatives from NSD, ANL, LBNL, and ORNL.)

Integration should be enlarged to include the more conventional (DOE/LBNL) definition, i.e. configuration management and interface control.

Safety standards and tolerances should be included in interface control documents.

Safety considerations should be included in the design reviews of the subsystems.

The risk management plan should follow the Molecular Foundry approach with impact assessment.

The project should establish a risk registry to catalog all risks.

\section{Recommendations}

\section{Detector and Electronics}

Detector contingency should be separated from the contingency for the rest of the project. The cost of the detectors is a large fraction of the total cost of the project. The detector contingency should be handled with a change in scope (i.e. reduced number of detectors), while the rest of the project consists of smaller elements that may be handled more conventionally. 
The collaboration should look carefully at the WBS breakdown and resource allowances for the electronics and trigger. The preamplifier and, to a lesser extent, the DSP board are already well prototyped. The prototype and/or pre-production for the preamplifier may not be necessary, or may be truncated. Some of the funds could be reallocated into contingency. The current contingency is very low.

\section{Data Acquisition and Computing}

The WBS is currently too detailed. WBS entries should be no shorter than 2-3 months. This will lead to less WBS bookkeeping and churning for GRETINA Management and make the WBS more easily and reliably reviewed.

Presentation of the FTE count should be reformatted. A resource-loaded Gant chart should make clear that the funded and redirected FTE fragments map cleanly onto realistic personnel profiles.

Internal consistency of diagrams, tables, and funding/FTE numbers should be reviewed.

Development from the functional prototypes of analysis software (hit decomposition and photon tracking) that exists now into mature production software (with appropriate calibrations and with the capability of running flawlessly in an on-line environment) will require a major effort. This effort should be recognized with its own box on the organization chart, and with significant attention in the planning and review process. The degree of effort required for this should be examined.

Current prototypes of crystal digitizer boards are based upon VME and are constructed with an older chip set. Realistic tests need to be done with a realistic combination of back-plane, chip set, fullyloaded crate (with sufficient hardware for three crystals), and with an updated sorting algorithm on the readout module, to ensure that the bandwidth of the back-plane is sufficient to handle the expected load. Risks in this area are:

a) Possible change of readout module to quad-CPU module.

b) Possible increase in number of assemblies (crates).

c) Possible change of back-plane technology (to cPCI).

Personnel who work for the duration of the project can contribute more efficiently, taking ownership for (and understanding context and history of) tasks. Every effort should be made to adjust FTE profiles to levels, which keep specific funded and redirected personnel on board for the entire duration of an activity. This may affect funding levels required by software development, as the sustained development effort should not fall below a critical mass.

The Decomposition Farm could be characterized as a mid-range system. Management of the nodes and processes on the Farm will not be trivial, and the continuous running of the Farm in real-time is a critical need for experiments. Farm performance measurements (mean time between failures, mean time to recover, etc.) and procedures for graceful recovery from failure and process life cycle management should be defined.

\section{Mechanical Systems}


A significant amount of beneficial effort has been expended in risk assessment and risk mitigation. Documentation should be generated that takes credit for this effort. The process of generating this documentation may point to areas that need more work. Credit should be taken for the successful completion of GAMMASPHERE, development work on the detectors and the R\&D effort in DAQ.

The project should move forward with the mechanical requirements and interface definition documents now.

There is a near term need for formal interface control and specification documentation. The specification list must be established and controlled. There must be a formal approach to handle design maturation (i.e. change control).

The existing schedule needs improvement in that it does not include the mechanical effort to formulate the specification of the detectors and for assurance of mechanical system to detector fit.

The project should consider a stripped down mechanical system implemented as soon as possible. This limited implementation could incorporate the hemisphere and a support structure, but with no automated translation/rotation initially. The deleted equipment could be used as scope contingency.

The two-year hiatus in the mechanical schedule may result in the loss of knowledge in this area. The knowledgeable engineer may not be available two years from now. The key features of the mechanical design and related issues should be documented with Engineering Notes to a level sufficient for an uninitiated engineer to follow. The documentation of GAMMASPHERE is inadequate for this purpose.

The schedule should be reviewed/revised and made consistent with realistic staffing levels (i.e. whole bodies over continuous periods).

The detectors are subject to mechanical damage during installation. Have provisions for installation tooling (estimated to be in the region of $\$ 100 \mathrm{k}$ for design and fabrication) been included in the detector subsystem?

A fourth (lower) change control level should be added for changes to the configuration that do not have significant cost or schedule impact.

\section{Project Management}

A manager from the Laboratory's Procurement Department should be brought on board as soon as possible to give the project advice on issues such as the following:

a) Preparing a sole source document

b) RFP vs. RFQ (what documents are needed)

c) Multiphase procurement commitment

DOE must be brought into the picture for any sole source of this magnitude. Furthermore, the project should enlist the aid of the DOE, the Engineering Division, and Lab Management in dealing with procurement-related issues. 
The contingency without the detector is at $22 \%$ with the detector is at $17 \%$. It is unlikely that the level of contingency for the present stage of the project will successfully pass a DOE review because it is too low and needs to be increased.

The project should define the descoping path for all subsystems, including thresholds.

The project should complete the primary criteria and function requirements and "flow down" the project system design requirements.

The project should develop interface documents.

The project should develop change control procedures for managing requirement changes (configuration control).

Project management should include requirements management, interface specification, integration, and integrated safety.

The project should refine and complete the PEP to define roles / responsibilities, method of accomplishment, and baseline control plan.

The project should identify an ES\&H point of contact to ensure compliance with EH\&S (with representatives from the electrical and mechanical safety committees). The Integrated Safety Document should be more transparent.

The project should request a funding profile that can reduce cost risk.

The project should perform a risk based contingency analysis.

The project should review the planned effort profile to make sure that it is realistic and consistent with available resources and the schedule.

The project should track OPC deliverables to ensure timely deliverables to the project.

The project should define project completion criteria (system integration) to be system assembly and test and not commissioning with beam. 


\section{Addendum of March 29, 2004 to the GRETINA Vetting Review of Nov. 4 \& 5, 2003.}

This addendum summarizes the progress made by the Gamma Ray Energy Tracking In-Beam Nuclear Array (GRETINA) Project on addressing issues raised during the Vetting Review held on Nov. 4 and 5 , 2003.

1. Since the vetting review, GRETINA has passed DOEs Critical Decision 1 Review. As a consequence of this review, DOE acknowledged that GRETINA was under funded, mainly due to the Dollar/Euro exchange ratio that fell steeply in the last year, an issue that was also raised in the Vetting Review. DOEs advice was to use an exchange rate of 1 Euro to 1.20 Dollars for detector modules, with a $20 \%$ contingency for detectors. This has been done, and together with other changes requested by DOE, it caused the GRETINA total estimated cost to increase from $\$ 14.8 \mathrm{M}$ to \$17.0M.

2. Given that more funds were made available, the DOE review over-ruled the vetting review and requested that GRETINA should delete any reference to scope contingency from the Project Execution Plan (PEP). Any change of scope should be dealt with the normal change control procedures described in the PEP.

3. Before the Vetting review, the overall contingency for GRETINA was below $20 \%$. GRETINA acknowledged that the preamplifier and the DSP board are already well prototyped and decided to drop the pre-production phase of these modules. Also, with other changes in scope, the overall contingency was increased to $23 \%$, with a detector contingency of approximately $20 \%$ and with the rest of GRETINA at $25 \%$ contingency.

4. The GRETINA team revisited the Major Item of Equipment (MIE) schedule, and removed the embedded Research and Development (R\&D) portion that made it somewhat confusing and difficult to follow. Also, the staffing plan was revisited and the FTE profiles adjusted to levels that kept specific funded personnel for the entire duration of an activity.

5. The schedule of the mechanical systems portion of GRETINA was changed to start early in the MIE to better match DOEs funding profile. Mechanical detector installation tooling was included in the cost estimate and schedule.

6. All tables, diagrams, funding and FTE numbers were reviewed and made consistent between the PEP and the GRETINA Conceptual Design Report.

7. GRETINA will receive the first detector module prototype in the next few weeks. GRETINA is preparing a test stand that can better address the performance of a portion of the data acquisition and computing system and to test the software tracking and reconstruction performance and availability.

8. The GRETINA team started the definition of the requirement documents for all major GRETINA subsystems and the detailed specification of the modules and parts will follow. 
9. The management roles and responsibilities, as well as the MIE completion criteria, were reviewed and better defined. System integration is now called System Assembly and it includes installation and testing at LBNL, but not the full commissioning of the MIE. The change control procedure was reviewed and upgraded.

10. The LBNL procurement group has been contacted, and they assigned a collaborator to support GRETINA on the purchase mechanism of the detector modules.

11. GRETINA also identified an ES\&H point of contact to ensure compliance with EH\&S requirements. The ES\&H point of contact also supported GRETINA with the writing of the EH\&S-related portions of the PEP for the DOE review. 